\title{
Taking responsibility for the early assessment and treatment of patients with musculoskeletal pain: a review and critical analysis
}

\author{
Nadine E Foster ${ }^{* 1}$, Jan Hartvigsen ${ }^{2,3}$ and Peter R Croft ${ }^{1}$
}

\begin{abstract}
Musculoskeletal pain is common across all populations and costly in terms of impact on the individual and, more generally, on society. In most health-care systems, the first person to see the patient with a musculoskeletal problem such as back pain is the general practitioner, and access to other professionals such as physiotherapists, chiropractors, or osteopaths is still either largely controlled by a traditional medical model of referral or left to self-referral by the patient. In this paper, we examine the arguments for the general practitioner-led model and consider the arguments, and underpinning evidence, for reconsidering who should take responsibility for the early assessment and treatment of patients with musculoskeletal problems.
\end{abstract}

\section{Introduction}

Musculoskeletal pain is common across all populations and costly in terms of impact on the individual and, more generally, on society. Musculoskeletal disorders have consistently been among the most commonly reported work-related illnesses since recording began. In the UK, an estimated 9.3 million working days were lost through these disorders in 2008-2009 [1]. In Denmark, one quarter of all health-related disability pensions are assigned because of musculoskeletal disorders, and a Dane can look forward to, on average, seven years with poorer quality of life because of musculoskeletal-related pain and disability [2].

The most common musculoskeletal pain problems are low back pain, shoulder pain, neck pain, and knee pain, followed by widespread pain [3]. Given projected increases

${ }^{*}$ Correspondence: n.foster@keele.ac.uk

${ }^{1}$ Arthritis Research UK Primary Care Centre, Keele University, Keele, Staffordshire, ST5 5BG, UK

Full list of author information is available at the end of the article in the numbers and proportions of older people in the population, the impact of these problems and the demand for musculoskeletal medicine are set to rise [4]. Patients with musculoskeletal problems require access to effective and timely advice, assessment, and treatment services that enable them to fulfill their optimum health potential and remain independent.

Studies have shown that approximately $30 \%$ to $40 \%$ of individuals with musculoskeletal complaints will consult their general practitioner (GP) about the pain [3]. Others have confirmed the burden, in general practice, of common musculoskeletal pain, suggesting that it is the second leading reason for consultation, accounting for up to $30 \%$ of GP consultations [4]. For example, low back pain leads to approximately 7 million GP consultations per year in the UK [5] and is the second leading symptomatic cause for physician visits in the US, and, in Denmark, a family doctor sees, on average, at least one back pain patient per workday [6]. In most health-care systems, the first person to see the patient with a musculoskeletal problem such as back pain is the GP, and access to other professionals such as physiotherapists is still controlled largely by a traditional medical model of referral. For example, 23\% (1.6 million) of the total annual low back pain consultations in general practice in the UK result in onward referral [7], and 6.7 million musculoskeletal patients are referred each year to physiotherapy [8]. However, many patients seek care directly from health-care professionals other than their family doctor [6,9]; for example, at least one third of back pain patients in Denmark now choose to see a chiropractor as their entry into the health-care system [6], and 7.7 million adults in the US visited a chiropractor in the year 2000 [10]. There is evidence that this trend is increasing; from 2000 to 2003 , there was a $57 \%$ increase in US adults visiting a chiropractor [10], and from 2006 to 2010, the proportion of patients self-referring to physiotherapists in The Netherlands rose from $22 \%$ to $43 \%$ [11]. This raises the question of whether the current GP-led primary-care model for patients with musculoskeletal disorders and back pain is the best approach. Alternative options 
include transferring first-contact care to other professional groups (such as chiropractors, physiotherapists, and osteopaths) whose clinical interests and expertise more clearly focus on musculoskeletal problems, increasing and improving the training pathways of GPs or other medical doctors with musculoskeletal special interests or introducing clearer multidisciplinary care models in which a variety of professionals work together to share the responsibility for the early assessment and management of patients with musculoskeletal problems. In this paper, we examine the arguments for the GP-led model and consider the arguments, and underpinning evidence, for reconsidering who should take responsibility for the early assessment and treatment of patients with musculoskeletal problems.

\section{Arguments for retaining the general practitioner-led model of care \\ Identifying serious pathology}

One of the most common arguments for the GP-led model of primary care for musculoskeletal pain problems centers on the importance of the GP in identifying serious pathology or so-called 'red flag' indicators of possible serious pathology [12] and ensuring urgent referral for those cases. Patients and GPs may be concerned about changing the model of care for musculoskeletal problems given the argument that the doctor is best placed to identify serious causes of musculoskeletal pain such as tumor, fracture, or infection by paying close attention to these red flags - clinical signs that supposedly indicate serious pathology requiring further diagnostic investigations or immediate medical attention. Fears about missed pathology have led some to question whether other health professionals, such as physiotherapists, have sufficient knowledge of diagnostic strategies $[13,14]$. Understandably, many patients are concerned that their musculoskeletal problem may signify a serious or progressive disease that, if treated early, can be cured. Research, however, suggests that serious disease is rarely the case in common musculoskeletal presentations. For example, in the most common musculoskeletal presentation, low back pain, the frequency of diagnoses of serious pathology is very low in patients presenting in primary care. An inception cohort of 1,172 consecutive patients receiving primary care for acute low back pain in Sydney, Australia, demonstrated a very low prevalence of serious pathology, namely 11 cases $(0.9 \%)$, eight of which were vertebral fracture [15]. However, most patients in the Australian series (80.4\%) had at least one red flag, indicating that, when used in isolation, they have little diagnostic value in the primary-care setting [16]. Evidence suggests that concentration on differential diagnosis and red flags may even divert the GP from evidence-based practice and contribute to unnecessary investigations, over-medicalization, and increased disability and costs [17]. There is evidence that some relatively uncommon musculoskeletal conditions (notably rheumatoid arthritis, gout, and polymyalgia) benefit from early diagnosis and treatment, but there is also evidence of substantial variation in the quality of early GP diagnosis and treatment (for example, $[18,19]$ ) and this variation may be related to lack of evidence about diagnostic utility of early symptoms and signs. It seems reasonable to ask for evidence about the effectiveness of musculoskeletal therapists in referring patients who may have such early conditions before assuming that doctors do it better and that all musculoskeletal patients must therefore be seen first by a GP. Some red flags (such as weight loss) are common between musculoskeletal conditions and some are condition- or site-specific, highlighting the need for primary-care professionals to have adequate training in differential diagnosis and in spotting unusual presentations of patients. There is no evidence that GPs are better than other well-trained health-care professionals at spotting these rare cases. Rather, the evidence to date suggests that there is no difference in the accuracy of diagnoses reached by GPs and other professionals for musculoskeletal disorders [20]. This contrasts with GP management of conditions such as angina or diabetes, in which toolboxes of diagnostic and practical management skills have been acquired throughout training and in which GP interventions clearly make a difference.

\section{Complexity and multimorbidity}

A second argument focuses on the GP's role in the care of patients with multiple chronic conditions or multimorbidity in primary care. Multimorbidity is common in the population (58\%) and in people with back pain [21]; indeed, most consultations in primary care involve people with multimorbidity (78\%) [22]. These patients are likely to be more complex to assess and treat and are likely to proceed to poorer clinical outcomes over time. For example, the combination of chronic musculoskeletal pain and depression is associated with clinical outcomes that are worse than those of either condition alone [23]. Some argue that musculoskeletal practitioners such as physiotherapists or chiropractors do not have the breadth of knowledge across common multimorbidities to identify and manage these patients well. It is certainly true that the patient requiring medical management of a range of chronic conditions such as diabetes or coronary heart disease, both of which occur more frequently in persons with chronic musculoskeletal pain, expects and requires the attention of a medical practitioner. However, it is also true that, ironically for the patient who has multiple health problems and who places priority on their musculoskeletal problem, there is evidence that the GP will tend to place priority on the other health conditions 
rather than the musculoskeletal problem [24]. Furthermore, many of the general principles of long-term management of chronic health problems, such as education, support for self-care, and enhancing the individual's functional ability and quality of life despite disability, represent central tenets of physiotherapy, for example, and apply outside the field of musculoskeletal pain as well [25].

\section{The general practitioner as patient advocate in systems with gate-keeping services}

For up to 100 years in many countries such as the UK, Denmark, Norway, and Sweden [26], the GP has had a pivotal role in the referral of patients to medical specialists and many other health-care professionals. The core values of general practice include comprehensiveness of care, a focus on the person with the disease and their psychosocial context, continuity of care, and the doctorpatient relationship over time, and these encourage the GP to take responsibility for the whole patient, irrespective of the specific health problem [27]. It has been argued that most patients in such 'gate-keeping' systems of health care value having one point of initial contact with a health professional they know and trust when experiencing significant health concerns and that, if allowed to choose their primary care through direct access to specialists, patients often do not go to the right specialist, because they do not have the ability or confidence to select appropriate care [28]. Although the primary reason for introducing this principle of referral, or the 'gate-keeping role', was the protection of the income of GPs [26], some believe that it has proved to be a sensible and important way of regulating and coordinating primary and secondary health care [26], ensuring the cost-effectiveness of health services such as the National Health Service (NHS) in the UK [29] and the cost-effective delivery of health services more broadly [30]. The move to 'single issue' services such as those for diabetes, depression, or musculoskeletal conditions can be thought of as representing a 'cherry picking' approach to health care [27] that may not, in the longer term, lead to improvements in population health and risk diversification but rather risks increased diversification and fragmentation of primary care.

\section{General practitioners with special interest in musculoskeletal medicine}

Concerns about the long waiting times for consultant appointments following GP referral and claims that many referrals by GPs to specialists were inappropriate or unnecessary fuelled the call for, and development of, GPs with special interests (GPSIs). The emergence of GPSIs in some countries such as the UK and Denmark offers the potential for more care to be provided closer to home
[31] and for referrals to hospital consultants to be reduced. In 2004, there were approximately 1,300 GPSIs in the UK across a wide range of health conditions; by 2011, there were in the region of 3,000 to 4,500 GPSIs. Each of the 152 Primary Care Trusts in England, for example, has approximately 20 to 30 GPSIs across clinical fields such as dermatology and gynecology and, less commonly, rheumatology, pain, and musculoskeletal. There is great variation across the UK in terms of the GPSI role, job specification, qualifications, and governance arrangements. Any one Primary Care Trust may have only two or three GPSIs in musculoskeletal pain, and, although GPSIs clearly have the potential to enhance the primary-care management of patients with musculoskeletal conditions, their small numbers relative to patient demand means that GPSIs alone cannot provide a comprehensive solution in the UK. Similarly, in Denmark, the Society for Musculoskeletal Medicine lists fewer than 100 out of a total of around 3,500 GPs who are certified in musculoskeletal medicine.

\section{Arguments for considering other models of first-contact care for musculoskeletal patients}

Although there are clearly multiple arguments in support of retaining the GP-led model of primary care for patients with musculoskeletal pain, there are increasingly compelling arguments for rethinking who should take responsibility for first-contact musculoskeletal care. First supported by a study in the British Medical Journal over 20 years ago [32] is the drive for professionals other than the GP to act as first port-of-call for musculoskeletal problems. Such 'primary-care musculoskeletal specialists' could provide extended and consistent evidence-based management and hence optimize the opportunity for better clinical outcomes from current episodes of pain as well as better promote secondary prevention. The GP could then become involved in the care of the minority of patients with complex health problems or in the minority of patients needing a more extensive investigation. The arguments in favor of reconsidering the model of firstcontact care for patients with musculoskeletal problems include advantages to patients, to musculoskeletal therapists such as physiotherapists and chiropractors, and to GPs themselves.

\section{The potential for improved patient care}

One challenge for GPs is that the management of many musculoskeletal conditions in primary care is about symptoms and function, movement and rehabilitation, activity, and positive attitudes rather than the traditional medical model of diagnosis and medical treatment. Previous studies confirm that patients with common musculoskeletal conditions such as osteoarthritis or back pain report GPs 'not taking their complaint seriously' 
[33] and therefore patients are left with the message that 'there is nothing to be done'. It seems sensible, therefore, to consider whether professional groups who actively embrace evidence-based care of patients with these conditions and who are actively engaged in leading training and research in these fields should be 'keeping the gate' for people who seek care for musculoskeletal problems.

Current GP care for musculoskeletal conditions is variable, consisting mostly of medication, brief advice $[17,34]$, and relatively poor information about prognosis [35]. A recent Australian survey showed that usual care for patients with back pain does not, on average, match care endorsed by international, evidence-based guidelines [17] and that GPs favor expensive management strategies, including medication and imaging, over simpler and universally recommended treatments. Pathways of care for patients presenting with musculoskeletal pain are often chaotic [36], and GPs manage patients themselves or refer them to any one or more of several providers and agencies, ranging from interface services, telephone triage services, physiotherapy services, complementary and alternative medicine (CAM) practitioners, podiatry services, and pain management services to traditional hospital orthopedic and rheumatology services. Furthermore, there is evidence that many GPs lack an understanding of what musculoskeletal professionals such as physiotherapists or chiropractors can offer their patients [37] or the value of treatments (such as exercise) offered by these professionals [38]. Clinical guidelines for the management of common musculoskeletal problems such as back pain [5] and joint pain attributed to osteoarthritis $[39,40]$ recommend pharmacological treatments, physical treatments such as exercise, and (for those not responding well to these treatment options) considerations of psychological treatments or surgery or both. At present, patient care is fragmented, GPs mostly offer advice and medication, and onward referral to other professional groups determines access to additional treatments. Recent initiatives to widen medication-prescribing rights to allied health professionals, including physiotherapists [41], and evidence that such professionals can be up-skilled to deliver clinically effective and psychologically informed interventions using principles of cognitive-behavioral therapy $[42,43]$ highlight the possibilities, and benefits, of greater engagement of these professionals in the provision of best primary care. These developments may relieve a currently unnecessary burden on GPs who are asked simply to write a prescription for pain medication recommended by the physiotherapist.

In addition, there is evidence that early intervention in general, and early treatment by physiotherapists in particular, for common musculoskeletal problems such as low back pain can reduce the amount of time people are off sick and can help to prevent acute problems from becoming chronic [5,44-46]. Although early intensive treatment is not always beneficial for recovery in musculoskeletal injuries [47], services that ensure timely access to care for musculoskeletal pain contribute importantly to patient experience and satisfaction [48]. Furthermore, there is evidence that patients going directly to see musculoskeletal practitioners such as physiotherapists are not at risk of having their serious medical conditions overlooked [49] and that experienced physiotherapists have the same level of knowledge as orthopedic specialists [50,51], demonstrating good clinical diagnostic accuracy [52] and management decisions [53]. There is also evidence to suggest that models of care led by musculoskeletal professionals such as physiotherapists lead to fewer prescriptions and investigations, decreased need for expensive and invasive treatments [54,55], and fewer consultations back in the health-care system [56].

The best evidence from clinical trials indicates that primary-care treatments can achieve modest but definite improvements for patients with back pain. Encouraging people to stay active and at work, helping patients adjust their beliefs and expectations to realistic but achievable goals, and offering simple analgesia and a range of interventions such as exercise, manual therapy, and acupuncture as well as support for rehabilitation to the workplace should result in less suffering, disability, and missed work. These core treatments are consistently recommended in national and international guidelines [5,57] and are those that musculoskeletal practitioners such as physiotherapists, chiropractors, and osteopaths are specifically equipped to deliver. Many high-quality trials of different interventions test treatments versus a control group of usual primary care initiated by the GP; in most of these trials, the treatments are shown to be superior to such usual primary care [58], although the size of the average differences between groups is generally small to moderate. Cost-effectiveness data from randomized clinical trials indicate that primary care for patients with low back pain is not cost-effective unless it also involves one or more added components such as exercise, spinal manipulation, or behavioral counseling [59].

Clearly, it is important that the overall effectiveness and potential challenges of a front-line service run by primary-care musculoskeletal specialists be evaluated critically and that appropriate training, education, and development of practitioners to provide such a service be supported and maintained. Ferguson and colleagues [60] have highlighted the need for ongoing education of physiotherapists in the systematic recording of red flags. The evidence for specific interventions offered by musculoskeletal practitioners such as exercise and manual therapy varies from trial to trial; although the 
results of these trials may be attributed, at least in part, to the heterogeneous populations of patients recruited [58], the varying effectiveness of practitioners and their interventions needs to be considered also. The performance of such practitioners needs to be critically reviewed; there is evidence that many physiotherapists in the US, for example, may not be delivering guideline-based care [61] and that their counterparts in the UK may be working, as do many GPs, within more of a traditional biomedical model rather than an active behavioral model of rehabilitation [34]. However, our view is that the evidence to date provides good reason to suppose that a model in which front-line primary care is provided by a range of musculoskeletal professionals such as physiotherapists and chiropractors and in which GPs serve as a route of second-line referral will be just as safe and effective as the current GP-led model and provides sufficient reason to suppose that it could provide more appropriate, efficient, and effective care for most primary-care consulters with musculoskeletal problems. These suppositions, of course, need to be tested to estimate the costs and benefits of making a switch from the current model; the evidence to date supports the rationale for large-scale prospective evaluations of such service development and change, and pilot studies have shown that musculoskeletal practitioners such as chiropractors can, and are ready to, be included in national quality development systems [62].

\section{Meeting patient demand through improved choice for patients}

Greater freedom of choice in addition to improved and faster access to musculoskeletal care are further advantages for patients offered an open service to musculoskeletal practitioners $[13,63]$. This is important because the expected increased burden of musculoskeletal pain over the next 50 years means that current models of care need to be re-evaluated in order to provide musculoskeletal services that meet rising patient demand. Musculoskeletal pain has not yet been a national healthcare priority in most countries, but, given the aging population, the burden to society will continue to rise in the future [64].

Previous changes to musculoskeletal services have already devolved much care from hospital and specialist centers to primary care (for example, in the UK through the introduction of Clinical Assessment and Treatment (CAT) services [4] at the interface between primary and secondary care). Many of these CAT services employ health professionals such as physiotherapists to carry out initial patient triage and place the patient in the most appropriate pathway of care $[65,66]$. In Sweden, many orthopedic departments now use physiotherapists as front-line diagnosticians in triaging patients with osteoarthritis. In both countries, this has resulted in dramatic reductions in waiting lists for patients waiting to see rheumatologists [65] and surgeons [67] as well as good patient outcomes over time [66]. Patient choice suggests that this could be achieved in primary care. In Denmark, at least one third of patients with back pain now choose to see a chiropractor as their entry into the health-care system [6]; in the US, more than half of people who had suffered from back or neck pain during the past year had consulted an alternative health-care practitioner, most commonly a chiropractor or massage therapist, whereas only one third had been seen by a conventional provider [9].

There is growing evidence in favor of changing the GPled model of care. Self-referral to physiotherapists is well established in countries such as Australia, New Zealand, Canada, most states of the US, The Netherlands, and Scotland and in some services in England [68-70]. The Netherlands has operated direct access to physiotherapists since 2006, and an evaluation shows that this is particularly popular with younger patients, those with higher levels of education, those with the most common musculoskeletal complaints of back and neck pain, and those with recurrent pain problems [13]. In addition, data show increasing proportions of patients choosing to directly access physiotherapists, from $22 \%$ in the year of introduction of self-referral to $43 \%$ over the course of a 4-year period [11]. Direct access and freedom of choice about first-contact care for musculoskeletal problems thus clearly satisfy a need among patients. Evaluations of self-referral to physiotherapy have shown high patient satisfaction and have shown that GPs and physiotherapists strongly support having physiotherapists work at the first point of contact [13,71] for musculoskeletal problems. It also appears, from non-randomized studies, that self-referral to physiotherapy can be cost-effective [55]. Data on self-referral to physiotherapy in Scotland indicated that the average cost of an episode of care was $£ 66.31$ compared with $£ 88.99$ for a GP referral, suggesting an estimated cost benefit to NHS Scotland of $£ 2$ million per year [55]. Recent self-referral pilots across six Primary Care Trusts in England highlighted a reduction in the number of associated NHS costs, particularly for investigations and prescribing, and $75 \%$ of patients who self-referred did not require a prescription for medicines. Self-referral to physiotherapy did not lead to an increase in demand for services and led to reduced work absence among patients [72]. Evaluations of the introduction of self-referral to physiotherapy in The Netherlands showed that self-referring patients were treated in fewer treatment sessions (average of 2.3 fewer treatment sessions) and that overall there was no increase in the number of patients visiting a physiotherapist in comparison with the year before the introduction of self-referral [13]. In regard to chiropractors, patients have traditionally 
self-referred to chiropractors, and recent evidence suggests that chiropractors in the UK already view themselves as primary-contact practitioners within the musculoskeletal field [73]. Patient satisfaction for chiropractic treatment is high [74], and there is evidence that back pain patients treated by chiropractors incur lower costs (due mostly to less advanced imaging) than patients treated by GPs [75]. There is, however, also evidence suggesting that the characteristics of patients seeking care may not be comparable to those of patients seeking care from GPs $[6,54,71]$.

\section{Increased professional responsibility for allied health professionals}

In many countries, health-care professionals such as physiotherapists, chiropractors, osteopaths, and exercise therapists are in important positions to provide support for active self-management and positive treatment options - especially interventions related to exercise and prevention of future episodes - for patients with musculoskeletal conditions. Furthermore, there is emerging evidence that prevention and treatment of musculoskeletal problems in the aging population can be tied to prevention and treatment of other public health problems through the promotion of an active lifestyle and targeted exercise [76], an area in which primary-care performance is suboptimal [77].

Alternative models of care led by other health professionals are already well established in private practice and in many health services, including those in Australia, Denmark, and The Netherlands and some in the UK. Such services provide the opportunity for increased professional responsibility and challenge for musculoskeletal practitioners as they make their own decisions autonomously and in direct partnership with their patients. These professionals are interested and well educated in the diagnosis and management of musculoskeletal conditions and their care is associated with better clinical outcomes [45] and greater patient satisfaction $[6,74,78]$ and cost-effectiveness $[55,75,79]$ in comparison with GP care alone [59]. If patients first see these musculoskeletal practitioners, the majority are unlikely to require the input of GPs or secondary-care specialists [45] and those who do may be more likely to benefit from those consultations.

It is important to note again the lack of randomized controlled trials to assess the effectiveness of these new models of care. There are clear parallels with other services, however. Examples include oral health and dentistry and eye health and optometry, which provide well-established models of first-contact care for patients and from which patients with suspected serious or other pathology are then referred to their GP. Dentists are even licensed to perform surgery and have limited prescription rights. The potential benefits for musculoskeletal practitioners such as physiotherapists and chiropractors are considerable, but further evidence, preferably in the form of controlled clinical trial evidence about clinical and cost outcomes, is needed.

\section{Reducing the workload of general practitioners}

Changing the care pathways for patients with common musculoskeletal problems is expected to be met with resistance at the organizational level but is likely to be a relief to many GPs [80]. Direct access to musculoskeletal specialists may reduce the workload of GPs. For example, in the year of introduction of direct access to physiotherapy in The Netherlands, more than one fifth of all patients seen by physical therapists came via direct access and these were not a new group of patients [13] but were those with recurrent musculoskeletal problems who normally would have consulted their GP.

Traditionally, GPs receive little training in common musculoskeletal problems in undergraduate medical school, during medical internship, and in post-graduate education [81] and often have limited knowledge about the suite of non-pharmacological treatments available to patients. Surveys and interviews indicate a lack of confidence in examining and providing treatment to patients with back pain, and many GPs feel ill equipped, either relying on pharmacological management or subsequently referring patients to doctors with special qualifications or to physiotherapists, chiropractors, or osteopaths $[6,17$, 80]. This limited knowledge base contrasts starkly with that of musculoskeletal professionals such as physiotherapists, chiropractors, and osteopaths. Research and academic developments in the field of musculoskeletal pain are led by a range of health professionals, from physiotherapists and chiropractors to rheumatologists and psychologists, and musculoskeletal pain is a relatively neglected academic area for general practice despite its substantial impact on the workload of GPs. This means that much of the knowledge being generated about the assessment and management of musculoskeletal pain is seen as core business by those within physiotherapy and other musculoskeletal professional groups but rather less by the professional group (GPs) that most often provides front-line care for these patients.

\section{The future of front-line care for musculoskeletal patients}

At present, one can rightfully question whether physiotherapists, chiropractors, or osteopaths are capable of completely filling the role of primary-care provider for common musculoskeletal conditions and the extent to which their range of treatments have yet to establish a clear evidence base. Furthermore, important issues of improvements in basic training of these professions need 
to be addressed, and professional development and postgraduate education are required in varying degrees.

However, the important challenge is to develop a coherent health-care system that effectively deals with the prevention, treatment, and rehabilitation of musculoskeletal disorders and that involves all available resources and professions. Thus, the question we raise here is a practical one - whether to continue to organize primary care for musculoskeletal problems around GPs or to more clearly support physiotherapists, chiropractors, and osteopaths to increasingly move into first-contact care roles. We suggest that debate, evaluation, evidence, and gradual change rather than radical transformation are needed. One potentially fruitful path could be to integrate education of these professions with each other and also with the education of medical doctors so that professional barriers and suspicions could be broken down, a common language developed, and new and innovative strategies for practice created. Such integration of education has been ongoing for almost 20 years at one Faculty of Health Sciences in Denmark, where education of chiropractors is completely integrated in the education of medical doctors. Recently, government commissions in Norway and Sweden recommended that similar educational approaches be implemented and that they also include physiotherapy. This type of model ensures that musculoskeletal practitioners have a high level of diagnostic skills both inside and, importantly, outside of the musculoskeletal field [82].

Important questions of efficacy and cost-effectiveness of interventions offered by any professional group caring for patients with musculoskeletal pain remain to be researched and resolved, and there is evidence that a substantial proportion of patients can be managed effectively with minimal but nevertheless active intervention $[5,43]$. Non-medical professions are well accepted as primary-care providers of oral and dental health, visual health, and many aspects of mental health, and clinicians such as nurses and pharmacists have been shown to improve both quality and cost-effectiveness in the management of many conditions. We think it is time to debate and re-think the way front-line musculoskeletal care is delivered in our health services. The models we have reviewed and suggested need to incorporate and clarify the role of the GP, including providing appropriate medical care for those patients who are referred to them with potentially serious pathology, uncommon conditions requiring the care of a medical doctor, or complex medical care needs.

\section{Conclusions}

There are clear arguments for and against challenging the current GP-led primary-care model for patients with musculoskeletal pain. In our opinion, the projection of societal burden related to musculoskeletal conditions and recent research evidence about best care for these patients add considerable weight to the argument for working toward changing the current model. We propose that it is time to have this debate with openness, dialogue, and curiosity and to set aside professional tensions and traditional hierarchies. Although such tensions are understandable, focusing on them draws attention away from the primary goal, namely to improve the care of patients with musculoskeletal problems. Any change in care pathways will, of course, need to be closely examined in terms of patient experience, safety, and clinical and cost-effectiveness.

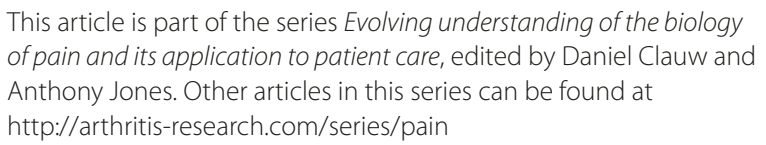

This article is part of the series Evolving understanding of the biology of pain and its application to patient care, edited by Daniel Clauw and Anthony Jones. Other articles in this series can be found at http://arthritis-research.com/series/pain

\section{Abbreviations}

CAT, Clinical Assessment and Treatment; GP, general practitioner; GPSI, general practitioner with special interests; NHS, National Health Service.

\section{Competing interests}

NEF is a physiotherapist who leads a program of primary-care intervention research focused on musculoskeletal pain. JH practiced as a chiropractor for 12 years before becoming a full-time academic. He currently leads a multidisciplinary research program in musculoskeletal health with a focus on spine pain at the University of Southern Denmark. PRC practiced as a family doctor for several years and continues to contribute to general practice education and research; he is a full-time researcher, working closely with GPs, rheumatologists, and therapists from a range of musculoskeletal disciplines.

\section{Authors' information}

NEF is a physiotherapist who leads a portfolio of intervention research for patients with common musculoskeletal pain problems in primary care. $\mathrm{JH}$ is a chiropractor who leads a comprehensive research program in musculoskeletal health at the University of Southern Denmark. PRC is a GP by background and epidemiologist by current practice.

\section{Author details}

'Arthritis Research UK Primary Care Centre, Keele University, Keele, Staffordshire, ST5 5BG, UK. ${ }^{2}$ Institute of Sports Science and Clinical Biomechanics, University of Southern Denmark, Campusvej 55, 5230 Odense M, Denmark. ${ }^{3}$ Nordic Institute of Chiropractic and Clinical Biomechanics, Forskerparken 10A, 5230 Odense M, Denmark.

Published: 29 February 2012

\section{References}

1. Health and Safety Executive: Musculoskeletal disorders (MSDs) in Great Britain (GB) [www.hse.gov.uk/statistics/causdis/musculoskeletal/index.htm].

2. Kjøller M, Kamper-Jørgensen F (eds): Public Health Report, Denmark 2007 [in Danish]. Copenhagen: Danish National Institute of Public Health, University of Southern Denmark; 2007.

3. Picavet HS, Schouten JS: Musculoskeletal pain in the Netherlands: prevalences, consequences and risk groups, the DMC3-study. Pain 2003, 102:167-178.

4. Department of Health: The Musculoskeletal Services Framework - A Joint Responsibility: Doing it Differently. London: Department of Health; July 2006.

5. Savigny $P$, Watson $P$, Underwood M: Early management of persistent nonspecific low back pain: summary of NICE guidance. BMJ 2009, 338:b1805.

6. Lønnberg F: The management of back problems among the population. I. Contact patterns and therapeutic routines [in Danish]. Ugeskr Laeger 1997, 159:2207-2214.

7. Rivero-Arias O, Gray A, Frost H, Lamb SE, Stewart-Brown S: Cost-utility 
analysis of physiotherapy treatment compared with physiotherapy advice in low back pain. Spine 2006, 31:1381-1387.

8. Chartered Society of Physiotherapy: A survey of NHS physiotherapy waiting times, workforce and caseloads in the UK, 2010-2011

[http://www.csp.org.uk/publications/

survey-nhs-physiotherapy-waiting-times-workforce-caseloads-uk-2010-2011].

9. Wolsko PM, Eisenberg DM, Davis RB, Kessler R, Phillips RS: Patterns and perceptions of care for treatment of back and neck pain: results of a national survey. Spine 2003, 28:292-297.

10. Davis MA, Sirovich BE, Weeks WB: Utilization and expenditures on chiropractic in the United States from 1997 to 2006. Health Serv Res 2010, 45:748-761.

11. Kooijman MK, Swinkels ICS, Leemrijse C, de Bakker D, Veenhof C: National Information Service of Allied Health Care [http://www.nivel.nl/oc2/page. asp?PagelD=9423\&path=/Startpunt/subsites/LIPZ\%202008/Fysiotherapie/ Wijze\%20van\%20toegang/Trends/Wijze\%20van\%20toegang].

12. Greenhalgh S, Selfe J: Red Flags: A Guide to Identifying Serious Pathology of the Spine. London: Churchill Livingstone Elsevier; 2006.

13. Leemrijse CJ, Swinkels IC, Veenhof C: Direct access to physical therapy in the Netherlands: results from the first year in community-based physical therapy. Phys Ther 2008, 88:936-946.

14. Deyle GD: Direct access physical therapy and diagnostic responsibility: the risk-to-benefit ratio. J Orthop Sports Phys Ther 2006, 36:632-634.

15. Henschke N, Maher CG, Refshauge KM, Herbert RD, Cumming RG, Bleasel J, York J, Das A, McAuley JH: Prevalence of and screening for serious spinal pathology in patients presenting to primary care settings with acute low back pain. Arthritis Rheum 2009, 60:3072-3080.

16. Underwood M: Diagnosing acute nonspecific low back pain: time to lower the red flags? Arthritis Rheum 2009, 60:2855-2857.

17. Williams CM, Maher CG, Hancock MJ, McAuley JH, McLachlan AJ, Britt H, Fahridin S, Harrison C, Latimer J: Low back pain and best practice care: a survey of general practice physicians. Arch Intern Med 2010, 170:271-277.

18. Roddy E, Zhang W, Doherty M: Concordance of the management of chronic gout in a UK primary-care population with the EULAR gout recommendations. Ann Rheum Dis 2007, 66:1311-1315.

19. Robinson PC, Taylor WJ: Time to treatment in rheumatoid arthritis: factors associated with time to treatment initiation and urgent triage assessment of general practitioner referrals. J Clin Rheumatol 2010, 16:267-273.

20. Patel S, Hossain FS, Colaco HB, El-Husseiny M, Lee MH: The accuracy of primary care teams in diagnosing disorders of the shoulder. J Eval Clin Pract 2011, 17:118-122.

21. Hartvigsen J, Frederiksen $\mathrm{H}$, Christensen $\mathrm{K}$ : Back pain remains a common symptom in old age. A population based study of 4,486 Danish twins ages 70-102. Eur Spine J 2003, 12:528-534

22. Salisbury C, Johnson L, Purdy S, Valderas JM, Montgomery AA: Epidemiology and impact of multimorbidity in primary care: a retrospective cohort study. Br J Gen Pract 2011, 61:e12-21.

23. Ang DC, Bair MJ, Damush TM, Wu J, Tu W, Kroenke K: Predictors of pain outcomes in patients with chronic musculoskeletal pain co-morbid with depression: results from a randomized controlled trial. Pain Med 2010 11:482-491.

24. Lugtenberg M, Zegers-van Schaick JM, Westert GP, Burgers JS: Why don't physicians adhere to guideline recommendations in practice? An analysis of barriers among Dutch general practitioners. Implement Sci 2009, 4:54.

25. Chartered Society of Physiotherapy: A career in physiotherapy [www.csp. org.uk/professional-union/careers-development/career-physiotherapy].

26. Loudon I: The principle of referral: the gatekeeping role of the GP. Br J Gen Pract 2008, 58:128-130

27. van Weel C: Reforming primary care: innovation or destruction. Br J Gen Pract 2012, 62:43-44

28. Shaw N, Hegedus G: The National Programme for Information Technology: the GP as gatekeeper--a bastion worth fighting for? Br J Gen Pract 2005, 55:85-86.

29. Salisbury C, Rosen R: Special branch: special interest GPs. Health Service Journal 1997, (July 19 suppl):14-16.

30. Squires D: International Profiles of Health Care Systems. New York: Commonwealth Fund; 2010.

31. Department of Health: The NHS Improvement Plan. Putting People at the Heart of Public Services. London: Department of Health; June 2004.

32. Hackett GI, Hudson MF, Wylie JB, Jackson AD, Small KM, Harrison P, O'Brien J, Harrison P: Evaluation of the efficacy and acceptability to patients of a physiotherapist working in a health centre. BMJ 1987, 294:24-26.

33. Alami S, Boutron I, Desjeux D, Hirschhorn M, Meric G, Rannou F, Poiraudeau S: Patients' and practitioners' views of knee osteoarthritis and its management: a qualitative interview study. PLoS One 2011, 6:e19634.

34. Bishop A, Foster NE, Thomas E, Hay EM: How does the self-reported clinical management of patients with low back pain relate to the attitudes and beliefs of health care practitioners? A survey of UK general practitioners and physiotherapists. Pain 2008, 135:187-195.

35. Mallen $\mathrm{CD}$, Peat $\mathrm{G}$ : Discussing prognosis with older people with musculoskeletal pain: a cross-sectional study in general practice. BMC Fam Pract 2009, 10:50

36. Ivanova Jl, Birnbaum HG, Schiller M, Kantor E, Johnstone BM, Swindle RW: Real-world practice patterns, health-care utilization, and costs in patients with low back pain: the long road to guideline-concordant care. Spine J 2011, 11:622-632.

37. Greene BR, Smith M, Allareddy V, Haas M: Referral patterns and attitudes of primary care physicians towards chiropractors. BMC Complement Altern Med 2006, 6:5.

38. Cottrell E, Roddy E, Foster NE: The attitudes, beliefs and behaviours of GPs regarding exercise for chronic knee pain: a systematic review. BMC Fam Pract 2010, 11:4.

39. Zhang W, Nuki G, Moskowitz RW, Abramson S, Altman RD, Arden NK, Bierma-Zeinstra S, Brandt KD, Croft P, Doherty M, Dougados M, Hochberg M, Hunter DJ, Kwoh K, Lohmander LS, Tugwell P: OARSI recommendations for the management of hip and knee osteoarthritis: part III: Changes in evidence following systematic cumulative update of research published through January 2009. Osteoarthritis Cartilage 2010, 18:476-499.

40. Conaghan PG, Dickson J, Grant RL; Guideline Development Group: Care and management of osteoarthritis in adults: summary of NICE guidance. BMJ 2008, 336:502-503

41. BBC News Health: Physiotherapy may get prescribing rights [http://www.bbc.co.uk/news/health-15037491].

42. Lamb SE, Hansen Z, Lall R, Castelnuovo E, Withers EJ, Nichols V, Potter R, Underwood MR; Back Skills Training Trial investigators: Group cognitive behavioural treatment for low-back pain in primary care: a randomised controlled trial and cost-effectiveness analysis. Lancet 2010, 375:916-923.

43. Hill JC, Whitehurst DG, Lewis M, Bryan S, Dunn KM, Foster NE, Konstantinou K, Main CJ, Mason E, Somerville S, Sowden G, Vohora K, Hay EM: Comparison of stratified primary care management for low back pain with current best practice (STarT Back): a randomised controlled trial. Lancet 2011, 378:1560-1571.

44. Wand BM, Bird C, McAuley JH, Doré CJ, MacDowell M, De Souza LH: Early intervention for the management of acute low back pain: a single-blind randomized controlled trial of biopsychosocial education, manual therapy and exercise. Spine 2004, 29:2350-2356.

45. Nordeman L, Nilsson B, Moller M, Gunnarsson R: Early access to physical therapy treatment for subacute low back pain in primary health care: a prospective randomized clinical trial. Clin J Pain 2006, 22:505-511.

46. Addley K, Burke C, McQuillan P: Impact of a direct access occupational physiotherapy treatment service. Occup Med (Lond) 2010, 60:651-653.

47. Côté P, Hogg-Johnson S, Cassidy JD, Carroll L, Frank JW, Bombardier C: Early aggressive care and delayed recovery from whiplash: isolated finding or reproducible result? Arthritis Rheum 2007, 15:861-868.

48. Xue CC, Zhang AL, Lin V, Myers R, Polus B, Story DF: Acupuncture, chiropractic and osteopathy use in Australia: a national population survey. BMC Public Health 2008, 8:105

49. Moore JH, McMillian DJ, Rosenthal MD, Weishaar MD: Risk determination for patients with direct access to physical therapy in military health care facilities. J Orthop Sports Phys Ther 2005, 35:674-678.

50. Weale AE, Bannister GC: Who should see orthopaedic outpatientsphysiotherapists or surgeons? Ann R Coll Surg Engl 1995, 77 (2 Suppl):71-73.

51. Childs JD, Whitman JM, Sizer PS, Pugia ML, Flynn TW, Delitto A: A description of physical therapists' knowledge in managing musculoskeletal conditions. BMC Musculoskelet Disord 2005, 6:32

52. Moore JH, Goss DL, Baxter RE, DeBerardino TM, Mansfield LT, Fellows DW, Taylor DC: Clinical diagnostic accuracy and magnetic resonance imaging of patients referred by physical therapists, orthopaedic surgeons, and non orthopaedic providers. J Orthop Sports Phys Ther 2005, 35:67-71.

53. Jette DU, Ardleigh K, Chandler K, McShea I: Decision-making ability of physical therapists: physical therapy intervention or medical referral. Phys Ther 2006, 86:1619-1629. 
54. Mitchell JM, de Lissovoy G: A comparison of resource use and cost in direct access versus physician referral episodes of physical therapy. Phys Ther 1997, 77:10-18.

55. Holdsworth LK, Webster VS, MCFadyen AK: What are the costs to NHS Scotland of self-referral to physiotherapy? Results of a national trial. Physiotherapy 2007, 93:311.

56. Hay EM, Foster NE, Thomas E, Peat G, Phelan M, Yates HE, Blenkinsopp A, Sim $\mathrm{J}$ : Effectiveness of community physiotherapy and enhanced pharmacy review for knee pain in people aged over 55 presenting to primary care: pragmatic randomised trial. BMJ 2006, 333:995.

57. Chou R, Qaseem A, Snow V, Casey D, Cross JT Jr., Shekelle P, Owens DK: Clinical Efficacy Assessment Subcommittee of the American College of Physicians; American College of Physicians; American Pain Society Low Back Pain Guidelines Panel: Diagnosis and treatment of low back pain: a joint clinical practice guideline from the American College of Physicians and the American Pain Society. Ann Intern Med 2007, 147:478-491.

58. Foster NE, Dziedzic KS, van der Windt DA, Fritz JM, Hay EM: Research priorities for non-pharmacological therapies for common musculoskeletal problems: nationally and internationally agreed recommendations. BMC Musculoskelet Disord 2009, 10:3

59. Lin CW, Haas M, Maher CG, Machado LA, van Tulder MW: Cost-effectiveness of general practice care for low back pain: a systematic review. Eur Spine J 2011, 20:1012-1023.

60. Ferguson F, Holdsworth L, Rafferty D: Low back pain and physiotherapy use of red flags: the evidence from Scotland. Physiotherapy 2010, 96:282-288

61. Fritz JM, Cleland JA, Brennan GP: Does adherence to the guideline recommendation for active treatments improve the quality of care for patients with acute low back pain delivered by physical therapists? Med Care 2007, 45:973-980.

62. Sorensen LP, Krog BR, Kongsted A, Bronfort G, Hartvigsen J: Development of disease-specific quality indicators for Danish chiropractic patients with low back pain. J Manipulative Physiol Ther 2011, 34:204-210.

63. Chartered Society of Physiotherapy: Self-Referral to Physiotherapy Services. London: Chartered Society of Physiotherapy; 2004.

64. Hartvigsen J, Christensen K: Back and neck pain are with us till the end. A nationwide interview-based survey of Danish 100-year olds. Spine 2008 33:909-913.

65. Maddison P, Jones J, Breslin A, Barton C, Fleur J, Lewis R, McSweeney L, Norgain C, Smith S, Thomas C, Tillson C: Improved access and targeting of musculoskeletal services in northwest Wales: targeted early access to musculoskeletal services (TEAMS) programme. BMJ 2004, 329:1325-1327.

66. Sephton R, Hough E, Roberts SA, Oldham J: Evaluation of a primary care musculoskeletal clinical assessment service: a preliminary study. Physiotherapy 2010, 96:296-302

67. Nation C: Physiotherapy and GP musculoskeletal interface clinics in Somerset. Making physiotherapy count. A range of quality assured services. London: Chartered Society of Physiotherapy; 2004:34-35 [http://www.csp.org.uk/uploads/documents/csp_making_physiotherapy_ count.pdf].
68. Galley P: Physiotherapists as first contact practitioners: new challenges and responsibilities in Australia. Physiotherapy 1977, 63:246-248.

69. Massey BF: American Physical Therapy Association Presidential address: What's all the fuss about direct access? Phys Ther 2002, 82:1120-1123.

70. Holdsworth LK, Webster VS: Direct access to physiotherapy in primary care: now and into the future? Physiotherapy 2004, 90:64-72.

71. Holdsworth LK, Webster VS, McFadyen AK: Are patients who refer themselves to physiotherapy different than those referred by GPs? Results of a national trial. Physiotherapy 2006, 92:26-33.

72. Department of Health: Self-Referral Pilots to Musculoskeletal Physiotherapy and the Implications of Improving Access to Other AHP Services. Leeds: Department of Health; 2008 [http://www.dh.gov.uk/en/Publicationsandstatistics/ Publications/PublicationsPolicyAndGuidance/DH_089516].

73. Jones-Harris AR: Are chiropractors in the UK primary healthcare or primary contact physicians? A mixed methods study. Chiropr Osteopat 2010, 18:28.

74. Butler RJ, Johnson WG: Satisfaction with low back pain care. Spine J 2008, 8:510-521.

75. Liliedahl RL, Finch MD, Axene DV, Goertz CM: Cost of care for common back pain conditions initiated with chiropractic doctor vs medical doctor/ doctor of osteopathy as first line physician: experience of one Tennesseebased general health insurer. J Manipulative Physio/ Ther 2010, 33:640-643.

76. Hartvigsen J, Christensen KL: Active lifestyle protects against incident low back pain in seniors. A population-based two-year prospective study of 1,387 Danish twins aged 70-100. Spine 2007, 32:76-81.

77. Armit CM, Brown WJ, Marshall AL, Ritchie CB, Trost SG, Green A, Bauman AE: Randomized trial of three strategies to promote physical activity in general practice. Prev Med 2009, 48:156-163.

78. Westmoreland JL, Williams NH, Wilkinson C, Wood F, Westmoreland A: Should your GP be an osteopath? Patient's view of an osteopathy clinic based in primary care. Complement Ther Med 2007, 15:121-127.

79. Williams NH, Edwards RT, Linck P, Muntz R, Hibbs R, Wilkinson C, Russell I, Russell D, Hounsome B: Cost-utility analysis of osteopathy in primary care: results from a pragmatic randomized controlled trial. Fam Pract 2004 21:643-650.

80. Breen A, Austin H, Campion-Smith C, Carr E, Mann E: 'You feel so hopeless': a qualitative study of GP management of acute back pain. Eur J Pain 2007, 11:21-29.

81. Chehade MJ, Burgess TA, Bentley DJ: Ensuring quality of care through implementation of a competency based musculoskeletal education framework. Arthritis Care Res 2010, 63:58-64.

82. Myburgh C, Mouton J: The development of contemporary chiropractic education in Denmark: an exploratory study. J Manipulative Physiol Ther 2008, 31:583-592.

doi:10.1186/ar3743

Cite this article as: Foster NE, et al:: Taking responsibility for the early assessment and treatment of patients with musculoskeletal pain: a review and critical analysis. Arthritis Research \& Therapy 2012, 14:205. 